\title{
Characterization of the nonlinear behaviour and the failure of human liver capsule through inflation tests
}

\author{
A. Brunon ${ }^{a, b, c, d, *}, K$. Bruyère-Garnier ${ }^{a, b, c}$, M. Coret $^{a, d}$ \\ a Université de Lyon, Lyon, F-69000, France \\ b Ifsttar, UMR_T9406, LBMC, F-69675, Bron, France \\ c Université Lyon 1, F-69622, Villeurbanne, France \\ d INSA-Lyon, LaMCoS UMR5259, F-69621, France
}

\begin{abstract}
This paper aims at describing an inflation test protocol on a human liver capsule using stereo-correlation. The biaxial tension created by the inflation test is comparable to the type of loading the capsule would be subjected to during a liver compression. Confocal microscopy associated to an anti-collagen coloration reveals that the tissue is isotropic at the meso-scale. Stereo-correlation provides the strain field of the capsule during the test. It emphasizes the boundary condition effects on the strain field. The measurement of the shape of the capsule is used to determine the parameters of two hyperelastic (polynomial and exponential) homogeneous models. The ultimate first principal strain before failure is measured locally and its value is $50.5 \% \pm 10.8 \%$. In this protocol, the light goes throughout the sample and makes the heterogeneities of the material appear as darker grey levels on the pictures. These heterogeneities also appear on the strain fields, so we can assume that they have different material properties.
\end{abstract}

\author{
Keywords: \\ Liver capsule \\ Inflation test \\ Stereo-correlation \\ Identification \\ Hyperelasticity \\ Failure
}

\section{Introduction}

Today, many road safety and vehicle regulations are based on validation tests like crash-tests. Such tests using anthropomorphic test devices do not give information about soft tissues and their behaviour under impact. Therefore, research turns towards numerical modelling to predict more precisely the occurrence of injuries on the different constituents of the human body, especially soft tissues. It is all the more necessary since various studies focused on accidentology revealed that damage to abdominal organs account for an important number of serious injuries in car accidents (Tinkoff et al., 2008; Welsh et al., 2007; Augenstein, 2000; Elhagediab and Rouhana, 1998). Liver damage in particular occurs in $38 \%$ of the injuries rated 3 to 5 in the Abdominal Injury Scale (AIS), AIS 5 resulting in a high risk of fatality.

Predicting liver injuries requires knowledge about its main constituents (vessels, parenchyma, capsule). Characterizing the constitutive laws and failure properties of these tissues is a first step in the numerical modelling of the whole organ. This study is focused on the liver capsule, which surrounds 
the liver. It is a thin connective tissue, mainly constituted of collagen fibres, which is involved in the external lesions of the liver.

Literature provides some data about abdominal organ connective tissues: some studies investigated their behaviour (Dan, 1999; Farshad et al., 1999) and failure properties (Herbert et al., 1976; Stingl et al., 2002; Snedeker et al., 2005) while others focused on the \{capsule, parenchyma\} material (Hollenstein et al., 2006; Valtorta and Mazza, 2006). They were based on global measurement of the forces and displacements and assumed the tissue to be homogeneous. In a previous paper (Brunon et al., 2010), Digital Image Correlation was used on uniaxial tensile tests conducted on human and porcine liver capsule and parenchyma samples, to determine locally the ultimate strain of the capsule. This method provides more accurate results than global measurements, as it computes the full local strain field and emphasizes any heterogeneity. In terms of failure, DIC is all the more necessary as failure is a localized phenomenon. However, using bimaterial samples to characterize the capsule created a high variability in the results. Therefore the capsule was chosen for independent study in this paper, after splitting it from the parenchyma (which was only possible for human liver but not for porcine liver).

Besides, characterizing nonlinear membranes is often conducted through inflation tests, on elastomers (Li et al., 2001; Johannknecht and Jerrams, 1999; Mott et al., 2003; Bhate and Kardos, 1984) or biological tissues (Bischoff et al., 2009; Schulze-Bauer et al., 2002). The identification of the constitutive parameters is conducted using either the external shape of the bubble, or the displacement at the pole, or displacement fields obtained by grid observation, associated to pressure measurements. Biaxial tests are shown to be necessary to correctly identify nonlinear behaviours (Johannknecht and Jerrams, 1999). Besides, in the case of failure characterization, the inflation test allows the loading of the tissue away from its edges, preventing the influence of possible failure initiations created during cutting.

Therefore, the present study aims at presenting an inflation test method, which allows the observation of material heterogeneities and the determination of a human liver capsule local strain failure criterion. An estimation of the parameters of a nonlinear constitutive law is conducted.

\section{Material \& methods}

\subsection{Preparation of the samples}

One human liver was removed from a Post-Mortem Human Subject (PMHS) from the Department of Anatomy of the University of Lyon through the French voluntary corpse donation to Science program. Capsule was taken off the liver using a syringe and a catheter to create a bubble of water between the capsule and the parenchyma; then the capsule was gently separated from the parenchyma into large pieces. The parts were kept at $4{ }^{\circ} \mathrm{C}$ until testing. $25 \mathrm{~mm}$ diameter circular samples were cut using a stamp and a scalpel. To be easily handled, each sample was put on a ring-shaped flat silicone seal. The samples were hydrated in a saline solution. Just before the test, a random pattern was applied on the sample with a paint spray (Fig. 1).

\subsection{Experimental device}

\subsubsection{Setup}

The capsule sample was fixed between two silicone flat seals on a plate made out of Plexiglas allowing pressure loading. This system was plugged into a compressed air general canal, through a pressure regulator and a lubricator (Fig. 2). The pressure regulator was used as a valve. The lubricator sprayed saline solution into the air flow to keep the capsule hydrated during the test, as recommended by Thornton et al. (2001) and Nicolle and Palierne (2010). The Plexiglas part allowed the light to go through the sample. Indeed, one of the main issues when using DIC on biological tissue is that hydration makes the tissue reflect the light and creates parasite pixels during the test, that prevent computing a good correlation. As hepatic capsule is very thin and translucent, this issue can be resolved by lightening through the sample (Fig. 2(c)). The video cameras therefore detect the shadow of the random pattern.

The silicone seals were particularly soft so as not to damage the capsule at its periphery. However, this softness caused the seals to lose their shape during the clamp tightening; the capsule buckled due to low bending stiffness. To compute the strain field during the test, the initial image for the DIC had to be taken when the tissue was already subjected to a small pressure causing it to tense. To identify this initial state, shape and curvature field were computed on the first images of the test (corresponding to "stereovision" in opposition to "stereo-correlation"), until the curvature was negative all over the sample (Fig. 3). Calculating the displacement fields from a buckled state would indeed lead to erroneous strain fields. The initial state corresponded to a deflection of around $1 \mathrm{~mm}$ at the pole of the sample and a pressure close to 0.02 bars.

The loading was applied by progressively opening the regulator to increase pressure gradually until the capsule failure. Post-processing revealed that the strain rate was approximately constant and corresponded to $10^{-2} / \mathrm{s}$.

\subsubsection{Measurements}

Two digital DALSA cameras with an image size of $1400 \times$ 1024 pixels $^{2}$ were used to capture the sample evolution and allow Digital Image Stereo-Correlation. During the test the field was a $25 \mathrm{~mm}$ diameter disk and the out-of-plane inflation of the capsule reached approximately $10 \mathrm{~mm}$. A $35 \mathrm{~mm}$ macro lens was therefore used on each camera to have the appropriate size and depth of field. The acquisition frequency was 50 frames per second.

The pressure acting on the capsule was measured by a 3bar ENTRAN EPX-N1 pressure sensor, connected to the PMMA plate. In what follows, the pressure referred to will be the relative pressure, that is the measured pressure subtracted the initial pressure measured at the initial state.

The displacement field on the capsule surface was determined using VIC3D ${ }^{\circledR}$ stereo-correlation software (Sutton et al., 2009). The calibration step gave the relative position of the two cameras. The stereo-correlation was conducted on each image to obtain the displacement field at every node of a $5 \mathrm{px}(0.18 \mathrm{~mm})$ grid, using $21 \times 21 \mathrm{px}^{2}\left(0.76 \times 0.76 \mathrm{~mm}^{2}\right)$ patterns. Then the software computed the strain field. The ultimate strain referred to in this paper is the first principal Green-Lagrange strain, computed by VIC3D ${ }^{\circledR}$. 
a

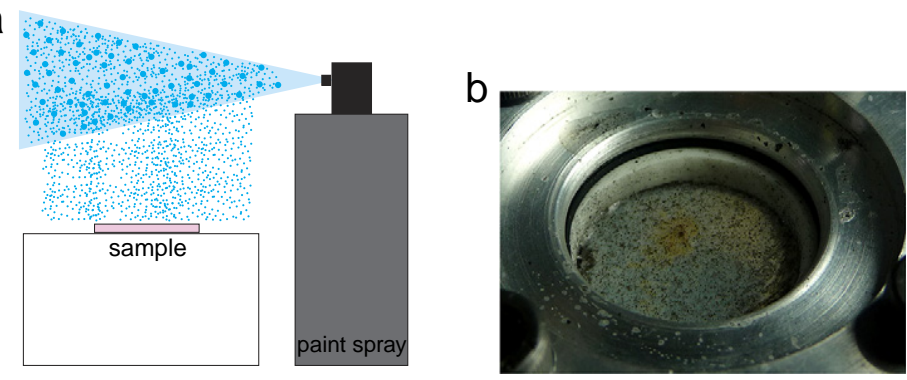

Fig. 1 - Spraying method for inflation test samples. (a) While spraying normally, gravity makes the small drops reach the sample. (b) Sample with random pattern fixed in the setup.
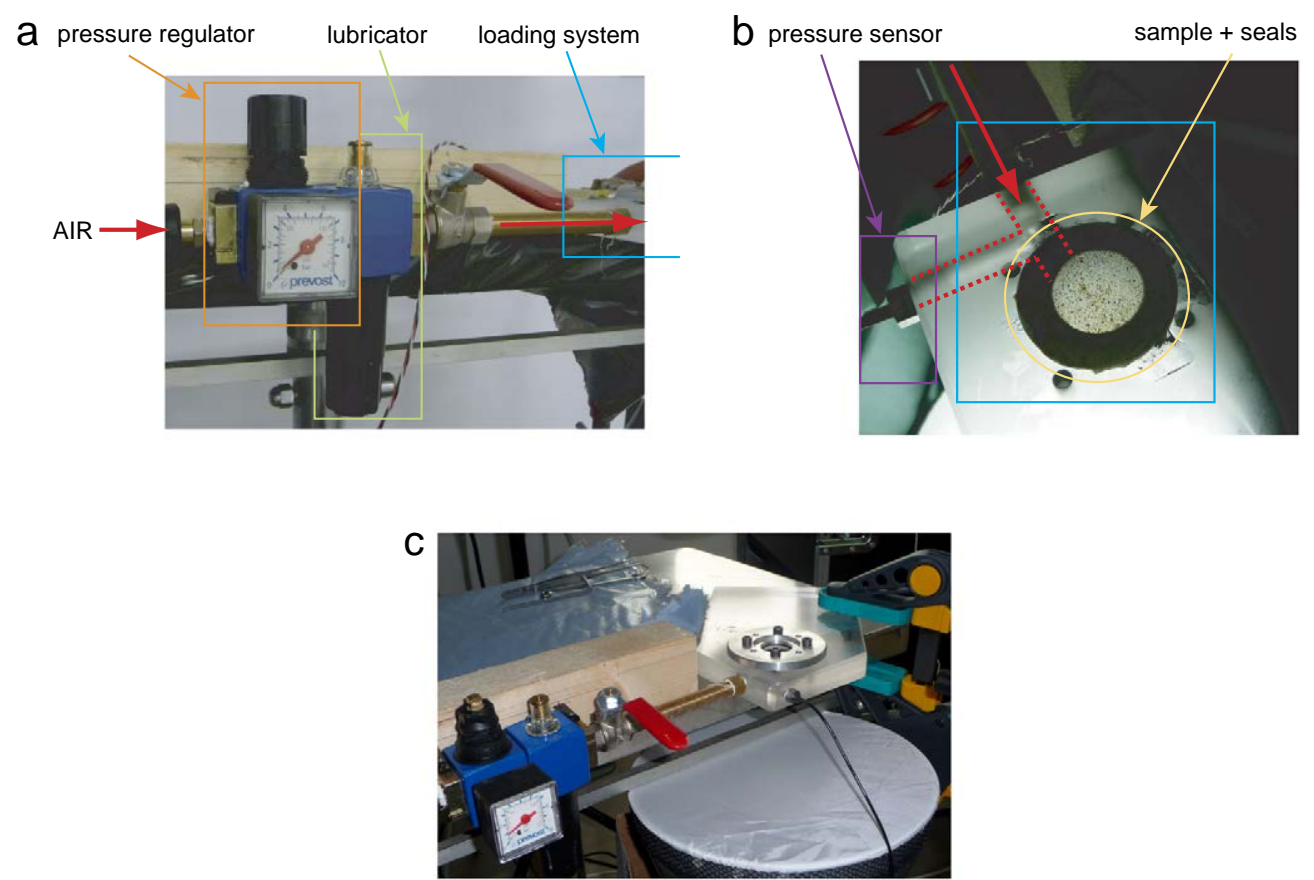

Fig. 2 - Experimental device: (a) Compressed air supply. (b) View of the dedicated system to load the sample (without the clamp and screws) when illuminated. (c) Global view; the light spot can be seen below the Plexiglas plate.

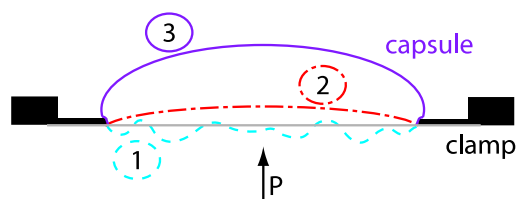

Fig. 3 - Determination of the initial state: (1) Buckled state of the capsule. (2) Initial state obtained by applying a small pressure: buckling has vanished, curvature is negative all over the sample and this state is close to a stress-free state. (3) Strained capsule.

\subsubsection{Confocal microscopy}

A Leica TCS-SP $2{ }^{\circledR}$ confocal microscope was used to observe the microstructure of the collagen in the capsule. Alexa 488 was used as an antibody to reveal the collagen fibres under blue laser light.

\subsection{Identification of the constitutive law}

The identification of the constitutive parameter of the capsule is conducted using the position of the capsule at each stereocorrelation step. At the initial state, the altitude $Z_{\min }$ of the lowest point of the area of interest is caught. At each step, the experimental position of the capsule is fitted by a sphere (radius $R$, center $O$ ). The section of the sphere and the horizontal plane at the altitude of $Z_{\min }$ is an approximation of the clamping circle, which radius is $r$ (Fig. 4).

The sample thickness is considered homogeneous and the capsule isotropic; $r$ and $R$ allow the determination of the radial and circumferential stretch ratios $\left(L_{r}, L_{c}\right)$, assuming that the initial state of the capsule is flat:

$L_{r}=\frac{\widehat{B C}}{A C}=\frac{\alpha \cdot R}{r}$

$L_{c}\left(d_{0}\right)=\frac{d}{d_{0}}=\frac{R}{d_{0}} \cdot \sin \left(\alpha \frac{d_{0}}{r}\right)$

with $\sin (\alpha)=\frac{r}{R}$. 


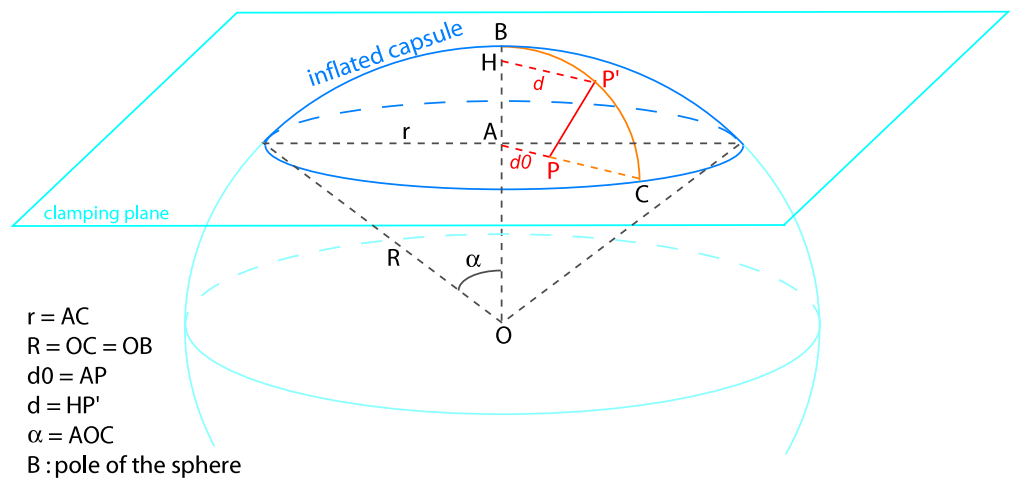

Fig. 4 - Analytical description of the inflated capsule: $P \in\{$ undeformed capsule $\}, P^{\prime} \in\{$ deformed capsule\}.

The vertical deflection at the pole of the sphere is around $1 \mathrm{~mm}$ at the initial state, corresponding to below $10 \%$ of the capsule radius, so the hypothesis of flat capsule is satisfactory. The expression of the radial and circumferential components of the first Piola-Kirchhoff stress tensor $\mathrm{T}_{1}$ and $\mathrm{T}_{2}$ once integrated through the capsule thickness is given in Erchiqui and Derdouri (2005):

$\mathrm{T}_{i}=\lambda_{i} \cdot \frac{\mathrm{dW}}{\mathrm{d} \lambda_{i}} \quad i=1,2$

with $\lambda_{i}$ the radial (1) and circumferential (2) stretch ratios, $\mathrm{W}\left(\mathrm{I}_{1}, \mathrm{I}_{2}\right)$ the strain energy potential of the capsule, and $I_{1}$ and $I_{2}$ the Cauchy-Green strain invariants. In this paper, two strain energy potentials are tested to fit the pressure/strain relationship: a particular form of the polynomial formulation $\left(\mathrm{W}_{\text {poly }}\right)$ and the exponential formulation $\left(\mathrm{W}_{\text {exp }}\right)$ :

$$
\begin{aligned}
W_{\text {poly }} & =\sum_{i+j=1}^{N} C_{i j}\left(I_{1}-3\right)^{i}\left(I_{2}-3\right)^{j} \\
& =C_{10}\left(I_{1}-3\right)+C_{20}\left(I_{1}-3\right)^{2}
\end{aligned}
$$

$\mathrm{W}_{\exp }=a \cdot \exp \left(b\left(I_{1}-3\right)-1\right)$

$I_{1}=\lambda_{1}^{2}+\lambda_{2}^{2}+\frac{1}{\lambda_{1}^{2} \cdot \lambda_{2}^{2}}$.

The constitutive laws (1) and (2) only involve $I_{1}$ because $I_{1}$ and $I_{2}$ are very close in the range of strain of this test (see next section). The exponential form (2) is proposed by Demiray (1972) to describe the behaviour of soft tissues, while the polynomial form (1) is a general description of hyperelasticity merging other usual laws (Neo-Hook, Mooney-Rivlin) in the case of comparable strain invariants.

The expression of the stress components is therefore: (polynomial)

$$
=\left\{\begin{array}{l}
\mathrm{T}_{1}=2\left(\frac{\lambda_{1}}{\lambda_{2}}-\frac{1}{\lambda_{1}^{3} \cdot \lambda_{2}^{3}}\right) \cdot\left(C_{10}+2 C_{20}\left(I_{1}-3\right)\right) \\
\mathrm{T}_{2}=2\left(\frac{\lambda_{2}}{\lambda_{1}}-\frac{1}{\lambda_{1}^{3} \cdot \lambda_{2}^{3}}\right) \cdot\left(C_{10}+2 C_{20}\left(I_{1}-3\right)\right)
\end{array}\right.
$$

(exponential)

$$
=\left\{\begin{array}{l}
\mathrm{T}_{1}=2 a b\left(\frac{\lambda_{1}}{\lambda_{2}}-\frac{1}{\lambda_{1}^{3} \cdot \lambda_{2}^{3}}\right) \cdot \exp \left(b\left(I_{1}-3\right)-1\right) \\
\mathrm{T}_{2}=2 a b\left(\frac{\lambda_{2}}{\lambda_{1}}-\frac{1}{\lambda_{1}^{3} \cdot \lambda_{2}^{3}}\right) \cdot \exp \left(b\left(I_{1}-3\right)-1\right) .
\end{array}\right.
$$

The equilibrium of the capsule leads to the relationship between the pressure under the capsule and the stress

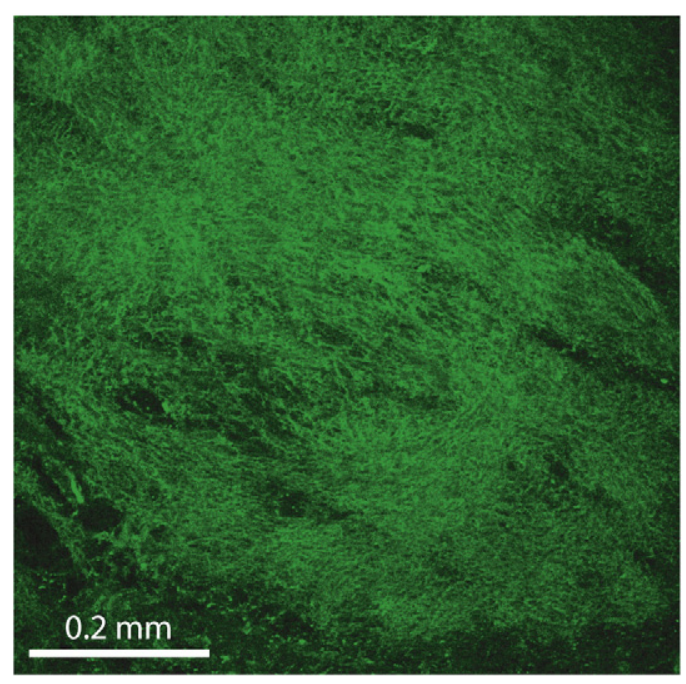

Fig. 5 - Observation of the collagen fibres of the capsule with a confocal microscope.

components:

$p=\frac{\mathrm{T}_{1}}{\rho_{1}}+\frac{\mathrm{T}_{2}}{\rho_{2}}$

with $\rho_{1}$ and $\rho_{2}$ the radii of curvature in the radial and circumferential directions. The inflated capsule is considered as a perfect sphere so $\rho_{1}=\rho_{2}=R$. The estimation of $p$ (3) can be computed at every correlation point. However, as described in Mott et al. (2003), the radial stretch ratio is constant along a radius while the circumferential one tends to one near the clamp. The calculation of $p$ will therefore be computed on the central zone of the area of interest, around the pole of the sphere, where $T_{1}$ and $T_{2}$ can be considered as homogeneous.

\section{Results}

\subsection{Confocal microscopy observations}

The image in Fig. 5 obtained with a confocal microscope shows that the collagen structure is isotropic: no strong directionality is detected. 

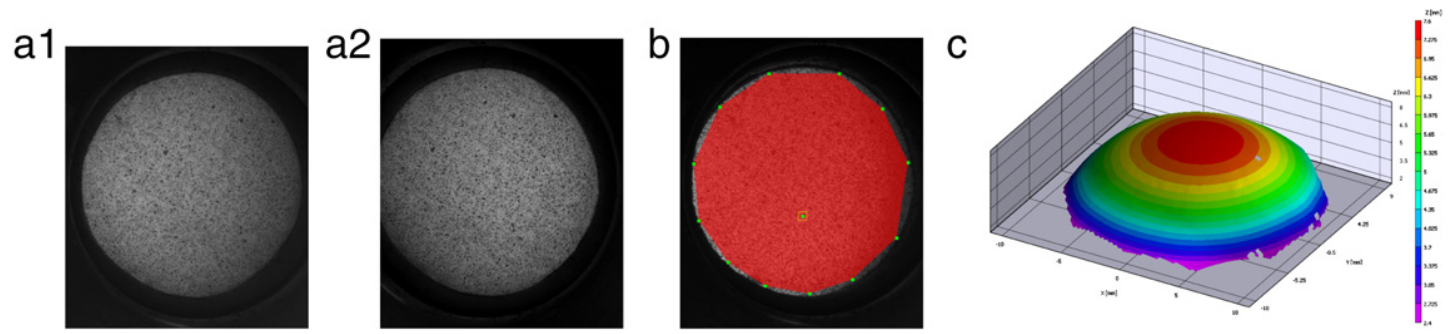

Fig. 6 - Capsule sample after pressure loading: (a1)-(a2) Deformed random pattern on the capsule recorded by the left and right camera respectively. (b) Zone Of Interest, where the stereo-correlation is computed. (c) Result of the correlation (altitude field in the cameras frame).

\subsection{Pressure and deflection evolutions}

During the test the capsule inflates until failure. An example of the shape of the capsule during its inflation is given in Fig. 6. For all the samples, the error on the displacement computed using DIC is below $0.1 \mathrm{px}$ corresponding to 3 . $10^{-3} \mathrm{~mm}$.

The evolution of the vertical displacement of the pole during the inflation test is given in Fig. 7. Tests \#6 and \#8 show an example of tests without failure, in which the sample slipped. The other tests show that the protocol is rather repeatable as the evolutions are similar between the tests.

Fig. 8 presents an example of the evolution of the shape of the capsule plotted in the (Y, Z) plane coinciding with the pole of the sphere, as the sample inflates.

\subsection{Constitutive parameter identification}

An example of the evolution of the experimental strain components along a radius of the sphere is plotted on Fig. 9. It is consistent with Mott et al. (2003): the circumferential component of the strain field tends to zero at the periphery and the radial component is rather constant from the pole to the periphery of the sample. It can be noted that the loading differs progressively from equibiaxial tension which would lead to identical radial and circumferential components around the pole of the sphere. It can be assumed that this difference is not due to anisotropy (as it does not occur from the beginning of the test and in accordance with the confocal microscopy observation) but to the boundary conditions, as the description of the strain fields will show. This graph shows that the radial and circumferential stretch ratios are generally constant until a radius of $100 \mathrm{px}$, corresponding to $4 \mathrm{~mm}$. The identification procedure is therefore conducted on a disk of $8 \mathrm{~mm}$ of diameter. Fitting the capsule with a sphere is satisfactory as the mean vertical distance between the perfect sphere and the experimental position of the capsule is $0.17 \pm 0.04 \mathrm{~mm}$, corresponding to less than $2 \%$ of the radius of the capsule. For both the polynomial and the exponential laws, the identification of the parameters consists in the least-square minimization of the gap between the experimental and the model pressure calculated using the strain components of the analytical model.

Fig. 10 justifies the restriction to one of the two strain invariants for the identification of the constitutive law of the capsule: the difference between $I_{1}$ and $I_{2}$ is below $20 \%$ in the range of strain of most of the samples.
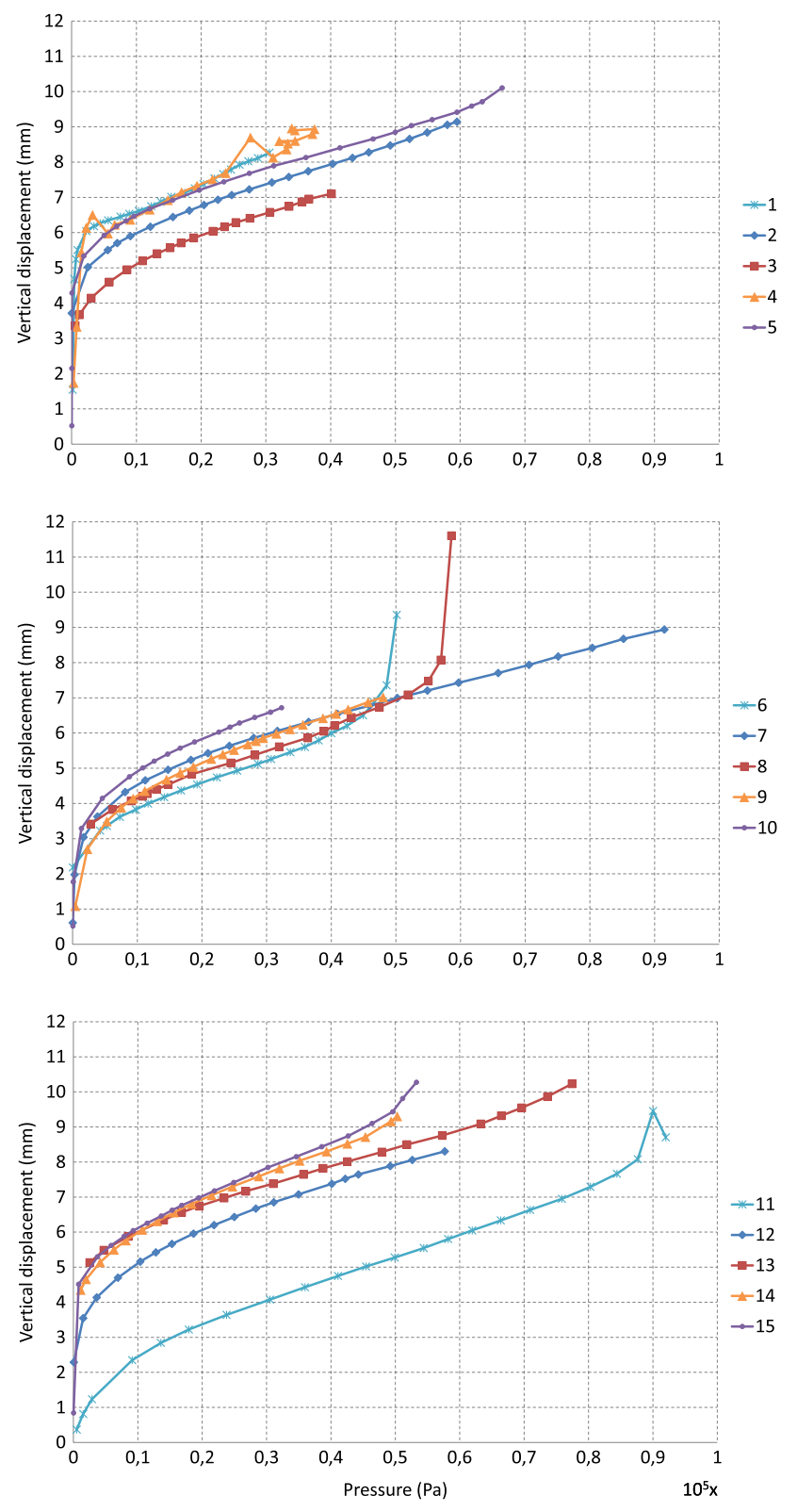

Fig. 7 - Evolution of the vertical displacement at the pole of the capsule submitted to an increasing pressure, for tests 1-15. 

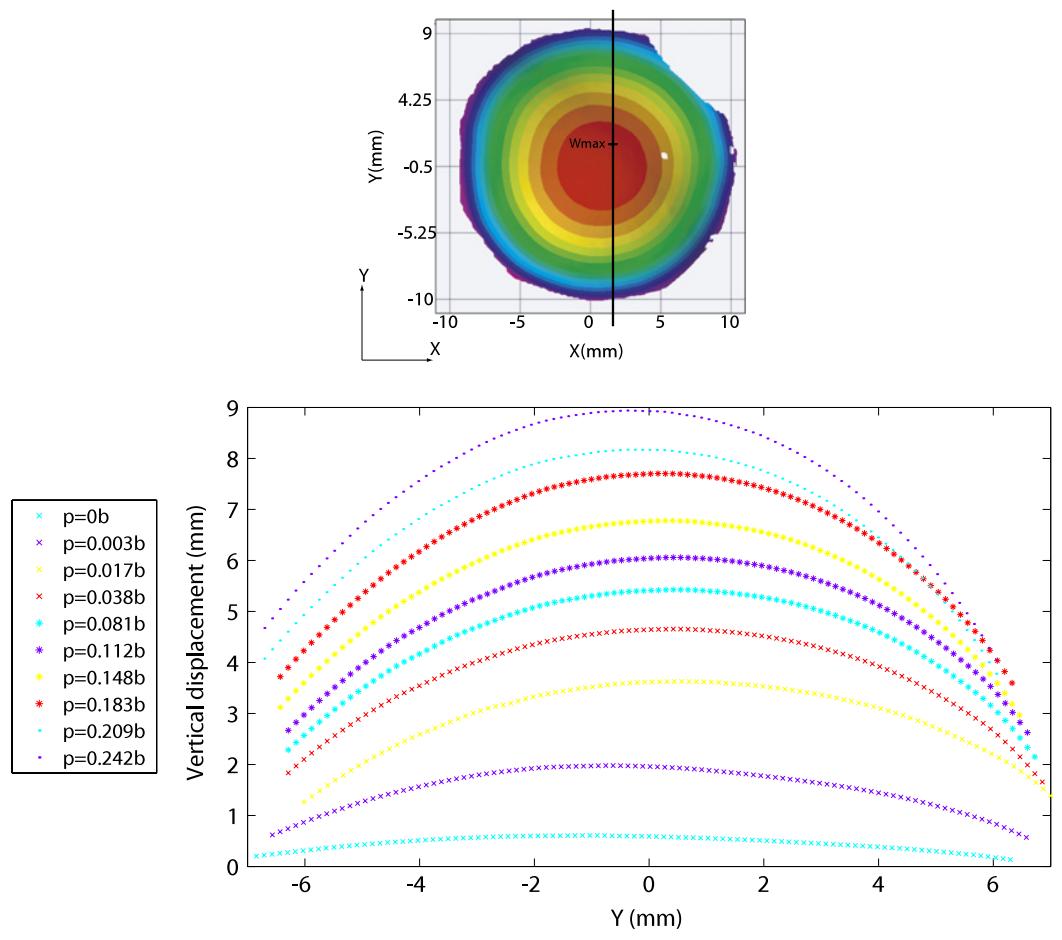

Fig. 8 - Example of evolution of the external shape of the capsule (test \#7): (1) Definition of the plot plane. (2) Vertical displacement versus abscissa for different pressure values in bars.

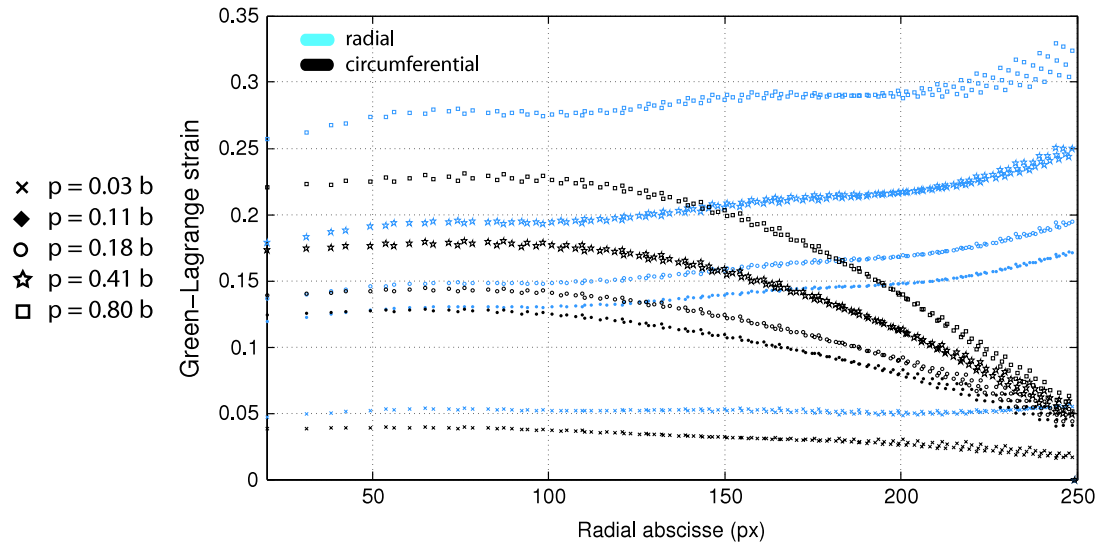

Fig. 9 - Example of radial and circumferential components of the strain field along a hemisphere radius on test \#7.

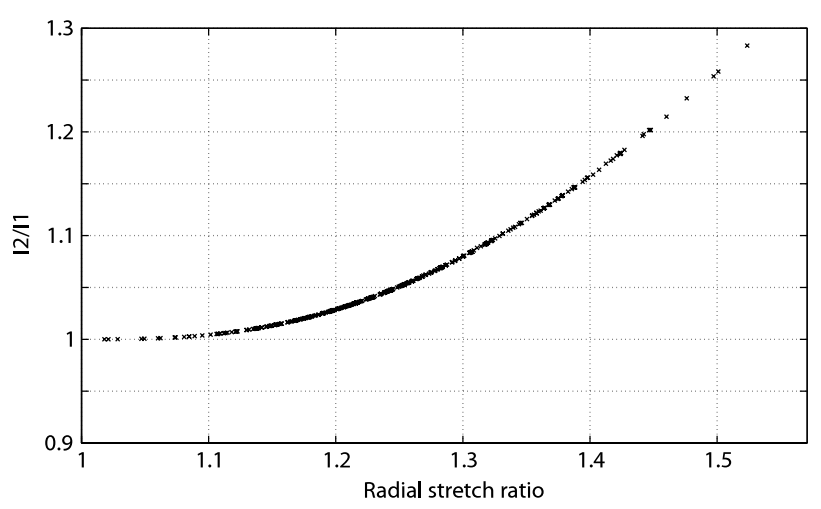

Fig. 10 - Ratio of invariants for the range of strains of this protocol.
A first identification of the two parameters of the polynomial law revealed that $C_{10}$ is very low or null. It was therefore decided to compute the identification of the following one-parameter polynomial law:

$\mathrm{W}=\mathrm{C}_{20}\left(\mathrm{I}_{1}-3\right)^{2}$.

The experimental curves and the identified laws, presented as corridors, are given in Fig. 11. The two models are similarly accurate: the mean error on the pressure is $0.13 \mathrm{bar}$ and 0.15 bar for the polynomial and the exponential models respectively, corresponding to $15 \%$ of the maximum pressure. The $C_{20}$ parameter value $\pm \mathrm{SD}$ is $114 \pm 40 \mathrm{kPa} \mathrm{mm}$ for the polynomial form; for the exponential law, $a$ and $b$ are respectively $71 \pm 29 \mathrm{kPa} \mathrm{mm}$ and $1.8 \pm 0.5$. It can be noted that the experimental curves exhibit a softening when reaching high levels 

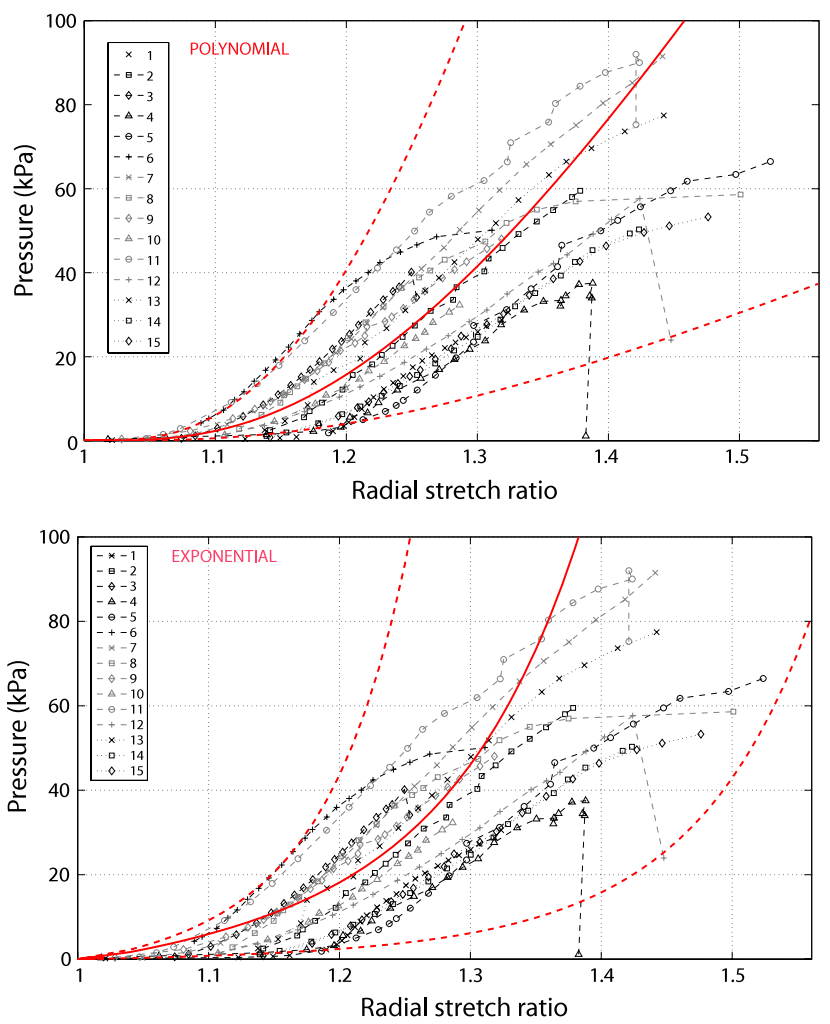

Fig. 11 - Identification of the constitutive law: experimental curves and polynomial law corridor. The corridor corresponds to: polynomial: $C_{20}=29 \mathrm{kPa} \mathrm{mm}$ (lowest curve), $C_{20}=294 \mathrm{kPa} / \mathrm{mm}$ (highest curve) and $\mathrm{C}_{20}=114 \mathrm{kPa} / \mathrm{mm}$ (mean curve)—exponential: $a=10 \mathrm{kPa} \mathrm{mm}, b=1.8$ (lowest), $a=50 \mathrm{kPa} \mathrm{mm}, b=3.5$ (highest), $a=75 \mathrm{kPa} \mathrm{mm}, b=1.8$ (mean).

of strain. The two models do not take into account this phenomenon which is not focused on in the present work.

\subsection{Description of the strain field}

Fig. 12 shows examples of strain fields that can be plotted after processing the DIC on an approximately homogeneous sample (no visible material heterogeneity). The vertical displacement field is made of generally circular isovalues, but when it comes to strains, the fields show heterogeneities: the Exx and Eyy should superimpose if subjected to a $90^{\circ}$ rotation, the principal strain fields E1 and E2 should be axisymmetric. This underlines the slipping occurring at the boundaries. In particular, the E1 and E2 fields reveal a cross shape that could be explained by the variability in the clamp tightening due to the use of 4 screws; it seems that buckling occurs in the tissue where it is less constrained. However, these differences between the expected and the experimental strain fields are captured by the DIC, which describes what actually happens during the test.

At the meso-scale, the strain field obtained by DIC allows the description of possible heterogeneities. Fig. 13 displays the principal strain fields of a sample with material heterogeneities (the darker areas on the picture (a)). The strain fields emphasize these heterogeneities (b1 \& b2), but as the correlation cannot be computed on them because of the low contrast, local information on their behaviour cannot be provided.

\subsection{Ultimate strain of the capsule}

The failure emerges where there is a defect that constitutes a stress concentration; the strain field consequently increases locally and rapidly (see an example of a failure initiation on Fig. 14). The setup did not prevent failure from emerging on the edge of the sample, but the ultimate strain could be measured anywhere on the sample by DIC. The failure occurs when the capsule is submitted to $0.59 \pm 0.17$ bar (number of samples: $n=10$ ). Among the samples that reached failure (in opposition to those that experienced slippage), the value of the ultimate Lagrange first principal strain is $50.5 \pm 10.8 \%$ $(n=10)$.

\section{Discussion}

The objective of the present study was to observe the human liver capsule response to an inflation test, corresponding to a more realistic loading than a uniaxial test. This methodology is pertinent because liver surface lacerations occur because of multiaxial loading on the liver surface.

The results of the Digital Image Correlation reveal that the problem of clamping complicates the optimization of the boundary conditions, that is achieving a circular and flat clamping of the capsule. Preventing sliding of the capsule during the test without damage prompted the choice of silicone seals, that makes the clamping shape differ from a flat circle and the capsule buckle. The cutting problem described in Brunon et al. (2010) is solved: the inflation test does not load the sample on its edges where cutting can create failure initiation. But the clamping creates other stress concentrations and strain localizations. Nevertheless, the full strain field clearly represents these kinematic phenomena.

Lighting throughout the sample guarantees that there are no reflexions on the capsule surface; the sample can therefore be correctly hydrated without compromising the image correlation. It also highlights the material heterogeneities at the meso-scale through a distribution of grey levels: it can be assumed that the darker levels correspond to more dense or thicker areas. The DIC shows that these heterogeneities have a clear influence on the strain field; however it could not be computed inside these particular areas, so a strong conclusion on their local behaviour cannot be drawn.

In this study, a hyperelastic constitutive law is identified using an existing model of inflated membrane. The hypothesis of isotropy used in this model is validated by confocal microscopy observations. The identification is based on the calculation of normalized loads corresponding to stresses integrated along the sample thickness; the constitutive parameters include therefore the thickness evolution during the inflation. The model pressure, to be compared with the experimental one, is calculated on the central disk of a sphere fitting the experimental position of the capsule; the hypothesis of perfect sphere is satisfactory as the mean vertical gap between the real and the perfect sphere is below $2 \%$ of the clamping radius. The evolution of 


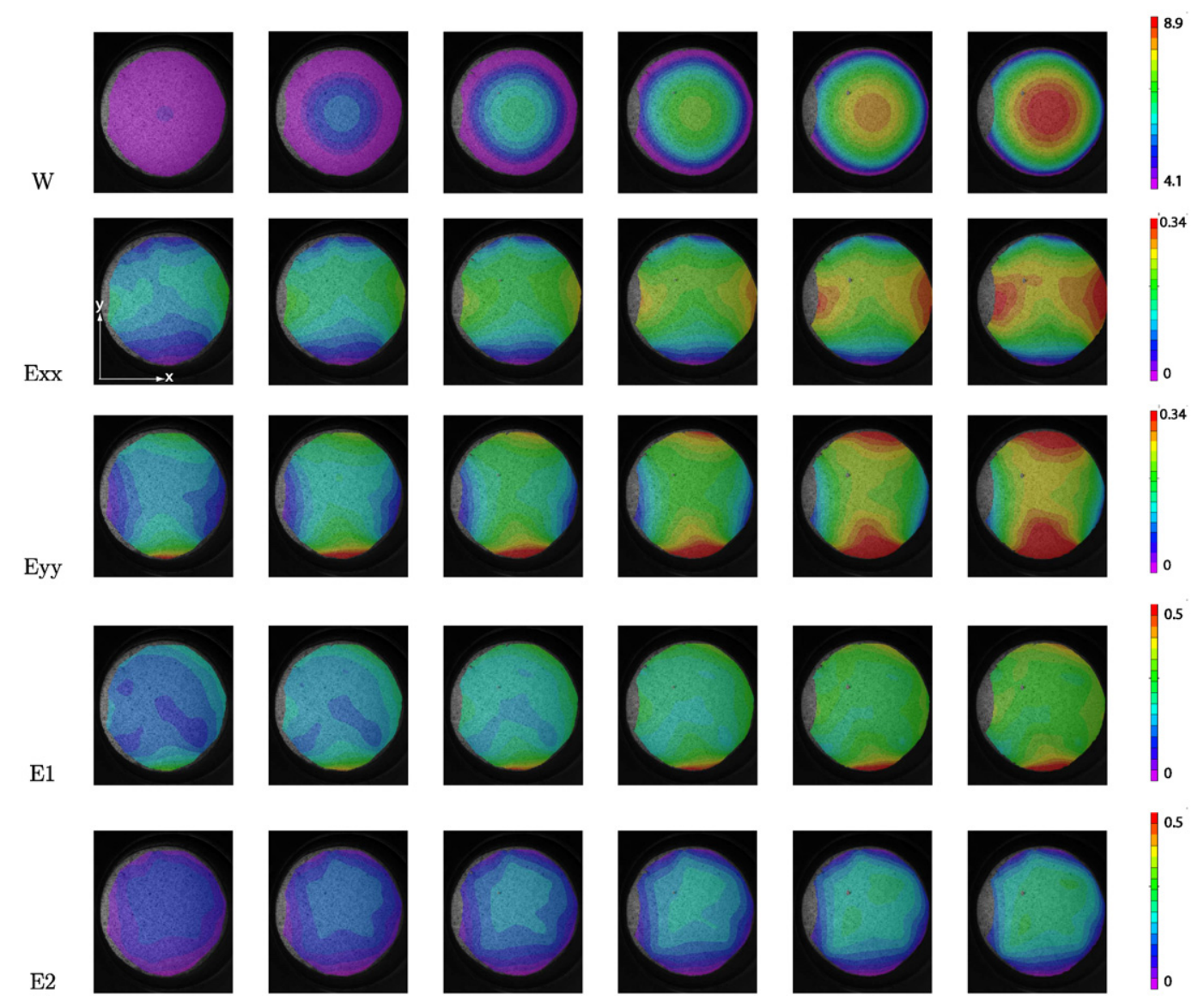

Fig. 12 - Example of fields obtained with the stereo-correlation (test \#7): W: vertical displacement (mm) - Exx, Eyy: Lagrange strains (on the picture: $x$-axis: horizontal - $y$-axis: vertical) - E1, E2: Lagrange principal strains.
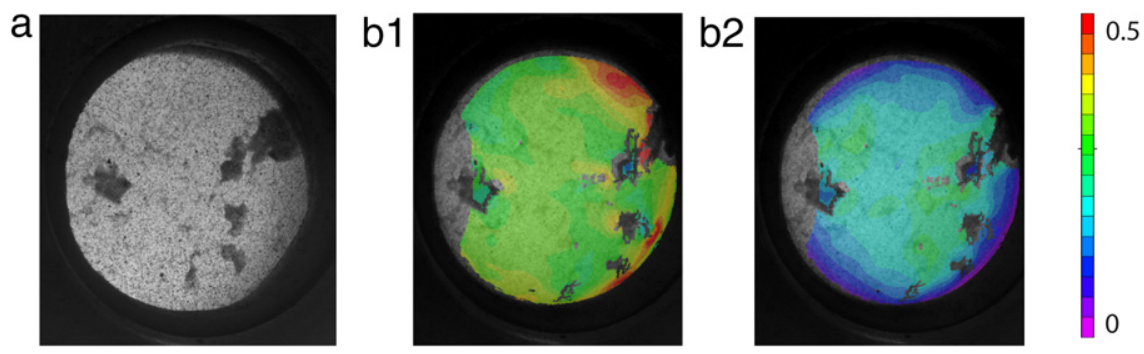

Fig. 13 - Strain fields before failure on a heterogeneous sample (test \#12): (a) Sample with the random pattern; large dark areas correspond to material heterogeneities. (b1) E1 Lagrange principal strain. (b2) E2 Lagrange principal strain.
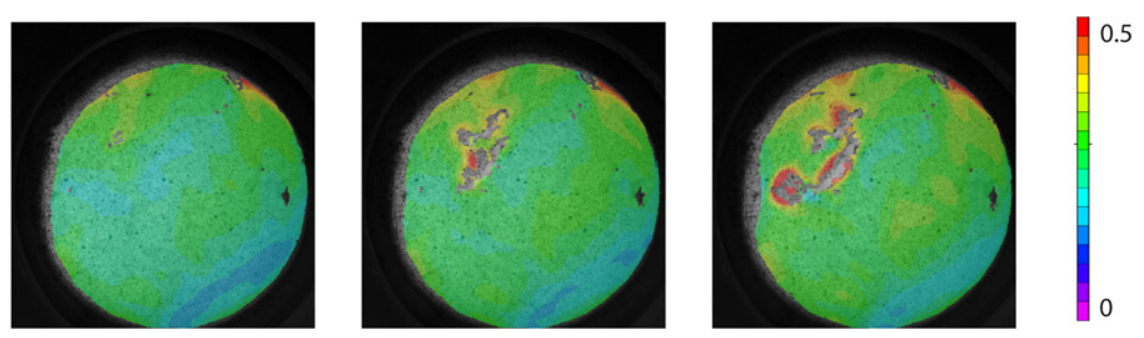

Fig. 14 - Emergence of a defect and localization of the strain until failure (E1 first principal strain fields) (test \#10). 
the pressure during the test can be well described by a oneparameter polynomial law or a two-parameters exponential law, both only involving the first strain invariant. It can be explained by the range of strain of this test (quasiequi-biaxiality and stretch ratios below 1.5) that makes the evolution of the first and second invariants very similar. The values of the constitutive parameters of both laws are consistent with the literature: in previous papers, slightly lower parameters were found for rat lungs (Schwenninger et al., 2010), slightly higher for human and rat tendons and human aorta (Pena et al., 2006; Calvo et al., 2010; Avril et al., 2010; Kroon and Holzapfel, 2008), similar for rat muscle and human cartilage (Calvo et al., 2010; Almeida and Spilker, 1998), which is consistent with the different collagen structures of those tissues. The limitation of the identification procedure conducted in this paper is that the sample thickness is generally heterogeneous, as it can be seen by lighting throughout the sample. The model is global and does not take into account these heterogeneities. Besides, the identification is conducted using the value of a unique scalar - the pressure - so the determination of multiple parameters can be unstable. It can be seen that the variability is higher for the two parameters of the exponential law than for the parameter of the polynomial law. Finally, the strain field description shows that the test is not perfectly axisymmetric and differs from an equibiaxial test, which is another hypothesis of the model.

The additional contribution of biaxial tests to the identification of constitutive parameters is described in Johannknecht and Jerrams (1999): the simulation of a multiaxial loading of a nonlinear material has to be conducted using stiffness parameters determined from biaxial tests. The simulation of an impact on a liver would lead to a multiaxial loading on the capsule, prompting the choice of biaxial tests for the further identification of the nonlinear constitutive law of the capsule. Depending on the number of parameters to identify, it could also be necessary to modify the setup so as not to subject the tissue to equi-biaxial loading but to any biaxial loading, so as to involve all the strain components in the identification procedure and to improve the identification accuracy. Using for instance an elliptic clamp creates a biaxial load with different values in each principal strain direction.

The failure criterion of $50.5 \pm 10.8 \%$ for the first principal strain determined in this study is based on ten measurements conducted on one liver. Its range is consistent with the values obtained previously in Farshad et al. (1999), Snedeker et al. (2005), Stingl et al. (2002), Hollenstein et al. (2006), Brunon et al. (2010). However, in spite of the large amount of samples, the values of constitutive parameters and ultimate strain determined in this paper must be taken as typical results rather than general values, as they do not take into account any biological variability between subjects.

Further work could consist in improving the identification of the constitutive law until failure of liver capsule. First, the nonlinearity of the tissue could be better described using additional biaxial tests and a Finite Element Analysis (Bischoff et al., 2009) for the identification of models with several parameters. It could be associated to the consideration of heterogeneities using a sub-domain identification (Seshaiyer and Humphrey, 2003). For a better resolution, using DIC on $2 \mathrm{D}$ and $3 \mathrm{D}$ tests combined with a nonlinear mechanical simulation, as described in (Réthoré, 2010) would allow building a specific heterogeneous model based on the grey level distribution of each backlighted sample and identifying local behaviour parameters of the capsule. Moreover, fully describing the behaviour of the tissue until failure requires the study of the softening part of the experimental curves. By taking into account the heterogeneities at the meso-scale and the softening at high strain levels, we could define a more accurate model of the capsule behaviour at the macro-scale, for an enhanced prediction of liver failure risk.

\section{Acknowledgements}

The authors would like to thank the Department of Anatomy of the University of Lyon and the French voluntary corpse donation to Science program; they also thank the Région Rhône-Alpes for its financial support.

\section{R E F E R E N C E S}

Almeida, E., Spilker, R., 1998. Finite element formulations for hyperelastic transversely isotropic biphasic soft tissues. Computer Methods in Applied Mechanics and Engineering 151 (3-4), 513-538.

Augenstein, J., 2000. Injury patterns in near-side collisions. In: SAE International World Conference.

Avril, S., Badel, P., Duprey, A., 2010. Anisotropic and hyperelastic identification of in vitro human arteries from full-field optical measurements. Journal of Biomechanics.

Bhate, A., Kardos, J., 1984. A novel technique for the determination of high frequency equibiaxial stress-deformation behavior of viscoelastic elastomers. Polymer Engineering and Science 24 (11), 862-868.

Bischoff, J.E., Drexler, E.S., Slifka, A.J., McCowan, C.N., 2009. Quantifying nonlinear anisotropic elastic material properties of biological tissue by use of membrane inflation. Computer Methods in Biomechanics and Biomedical Engineering 10 (3), 353-369.

Brunon, A., Bruyère-Garnier, K., Coret, M., 2010. Mechanical characterization of liver capsule through uniaxial quasi-static tensile tests until failure. Journal of Biomechanics 43 (11), 2221-2227.

Calvo, B., Ramírez, A., Alonso, A., Grasa, J., Soteras, F., Osta, R., Muñoz, M., 2010. Passive nonlinear elastic behaviour of skeletal muscle: experimental results and model formulation. Journal of Biomechanics 43 (2), 318-325.

Dan, D., 1999. Caractérisation mécanique du foie humain en situation de choc. Ph.D. Thesis, Université Paris 7.

Demiray, H., 1972. A note on the elasticity of soft biological tissues. Journal of Biomechanics 5 (3), 309-311.

Elhagediab, A., Rouhana, S., 1998. Patterns of abdominal injury in frontal automotive crashes. In: 16th International ESV Conference Proceedings. pp. 327-337.

Erchiqui, F., Derdouri, A., 2005. Analyse Expérimentale et Numérique du Comportement de Membranes Thermoplastiques en ABS et en HIPS dans le Procédé de Thermoformage. The Canadian Journal of Chemical Engineering 83 (3), 527-536.

Farshad, M., Barbezat, M., Flueler, P., Schmidlin, F., Graber, P., Niederer, P., 1999. Material characterization of the pig kidney in relation with the biomechanical analysis of renal trauma. Journal of Biomechanics 32 (4), 417-425. 
Herbert, L., Chen, W., Hartmann, A., Garancis, J., 1976. Mechanical properties of the dog renal capsule. Journal of Applied Physiology 40 (2), 164.

Hollenstein, M., Nava, A., Valtorta, D., Snedeker, J., Mazza, E., 2006. Mechanical Characterization of the Liver Capsule and Parenchyma. In: Lecture Notes in Computer Science, vol. 4072. pp. 150-158.

Johannknecht, R., Jerrams, S., 1999. The need for equi-biaxial testing to determine elastomeric material properties. In: Constitutive Models for Rubber: Proceedings of the First European Conference on Constitutive Models for Rubber. Taylor \& Francis, Vienna, Austria, p. 73.

Kroon, M., Holzapfel, G., 2008. A new constitutive model for multilayered collagenous tissues. Journal of Biomechanics 41 (12), 2766-2771.

Li, Y., Nemes, J., Derdouri, A., 2001. Membrane inflation of polymeric materials: experiments and finite element simulations. Polymer Engineering and Science 41 (8), 1399-1412.

Mott, P., Roland, C., Hassan, S., 2003. Strains in an inflated rubber sheet. Rubber Chemistry and Technology 76 (2), 326-333.

Nicolle, S., Palierne, J.-F., 2010. Dehydration effect on the mechanical behaviour of biological soft tissues: observations on kidney tissues. Journal of the Mechanical Behavior of Biomedical Materials 3 (8), 630-635.

Pena, E., Calvo, B., Martinez, M., Doblare, M., 2006. A threedimensional finite element analysis of the combined behavior of ligaments and menisci in the healthy human knee joint. Journal of Biomechanics 39 (9), 1686-1701.

Réthoré, J., 2010. A fully integrated noise robust strategy for the identification of constitutive laws from digital images. International Journal for Numerical Methods in Engineering 84 (6), 631-660.

Schulze-Bauer, C., Regitnig, P., Holzapfel, G., 2002. Mechanics of the human femoral adventitia including the high-pressure response. American Journal of Physiology-Heart and Circulatory Physiology 282 (6), H2427-H2440.
Schwenninger, D., Schumann, S., Guttmann, J., 2010. In vivo characterization of mechanical tissue properties of internal organs using endoscopic microscopy and inverse finite element analysis. Journal of Biomechanics doi:10.1016/j.jbiomech.2010.09.019.

Seshaiyer, P., Humphrey, J., 2003. A sub-domain inverse finite element characterization of hyperelastic membranes including soft tissues. Journal of Biomechanical Engineering 125, 363-371.

Snedeker, J., Niederer, P., Schmidlin, F., Farshad, M., Demetropoulos, C., Lee, J., Yang, K., 2005. Strain-rate dependent material properties of the porcine and human kidney capsule. Journal of Biomechanics 38 (5), 1011-1021.

Stingl, J., Baca, V., Cech, P., Kovanda, J., Kovandová, H., Mandys, V., Rejmontová, J., Sosna, B., 2002. Morphology and some biomechanical properties of human liver and spleen. Surgical and Radiologic Anatomy 24 (5), 285-289.

Sutton, M., Orteu, J., Schreier, H., 2009. Image Correlation for Shape, Motion and Deformation Measurements: Basic Concepts, Theory and Applications. Springer Verlag.

Thornton, G., Shrive, N., Frank, C., 2001. Altering ligament water content affects ligament pre-stress and creep behavior. Journal of Orthopaedic Research 19 (5), 845-851.

Tinkoff, G., Esposito, T.J., Reed, J., Kilgo, P., Fildes, J., Pasquale, M., Meredith, J.W., 2008. American association for the surgery of trauma organ injury scale i: spleen, liver, and kidney, validation based on the national trauma data bank. Journal of the American College of Surgeons 207 (5), 646-655.

Valtorta, D., Mazza, E., 2006. Measurement of rheological properties of soft biological tissue with a novel torsional resonator device. Rheologica Acta 45 (5), 677-692.

Welsh, R., Morris, A., Hassan, A., 2007. Struck-side crashes involving post-regulatory European passenger cars: crash characteristics and injury outcomes. International Journal of Vehicle Safety 2 (1), 103-115. 